\title{
EFFECTS OF CORRELATED GENE DISTRIBUTIONS IN THE ANALYSIS OF DIALLEL CROSSES
}

\author{
J. HILL
}

A.R.C. Unit of Biometrical Genetics, Department of Genetics, University of Birmingham

Received 2o.vi.63

\section{THE DIALLEL CROSS-ITS MERITS AND LIMITATIONS}

ThE diallel cross technique has in recent years become widely used experimentally, especially by plant breeders, to whom its chief value is that it enables a number of potentially useful breeding lines to be evaluated simultaneously. A reliable assessment of the relative values of the lines under test depends to a large extent upon the fulfilment of certain conditions which have previously been discussed in detail by Jinks and Hayman (1953) and Hayman (1958). Four of these conditions are general to all biometrical analyses, viz.,

(i) the mechanism of inheritance must be diploid,

(ii) maternal effects should be absent,

(iii) the parents to be used in the cross should be homozygous, and

(iv) there should be no interactions between genes at different loci.

In addition two further conditions must be satisfied in diallel crosses, viz.,

(v) no multiple alleles should be present, and

(vi) the genes must be randomly distributed among the parents.

If these assumptions can be proved valid for a particular diallel cross the results obtained from it can be interpreted in a comparatively clear manner. On the other hand, if analysis shows that they do not hold, difficulties in interpretation inevitably arise. Unfortunately, it is at this point that our knowledge of diallel crosses is at its weakest; the amount of experimental work which has so far been carried out to determine precisely what happens in the event of failure of one or more of these conditions is so limited as to preclude any definite conclusions from being drawn. The most extensive work on this problem is that of Jinks (1954, 1955), who was interested primarily in the effects of non-allelic interactions on the array covariance-variance graph. Whereas the regression line of $\mathrm{Wr}$ on $\mathrm{Vr}$ is normally expected to have a slope of I, Jinks found this slope to depart significantly from its expectation when gene interactions were present. Whilst non-allelic interactions are the most common source, they are by no means the sole cause of disturbance in the $\mathrm{Wr} / \mathrm{Vr}$ graph. Theoretically, it is possible to demonstrate that non-random gene distributions can 
generate characteristic upsets on the graph, but in practice the chief difficulty has been to obtain data which will display their effects in such a way as to enable them to be unambiguously separated from those of non-allelic interactions.

The present paper describes experiments with inbred lines of Drosophila melanogaster which were designed to simulate the effects of the two types of correlated gene distribution-association and dispersion. Furthermore it was hoped to obtain from these experiments information which would allow us to distinguish association on the one hand from dispersion on the other.

\section{THE EXPERIMENT}

Eight true-breeding lines of $D$. melanogaster, representing all possible combinations of chromosomes I, II and III from the two inbred stocks of Oregon (B) and Samarkand (S), were used to construct an $8 \times 8$ diallel cross. Details of the synthesis of these lines are given by Cooke and Mather (Ig62). The complete diallel was repeated on four different occasions with a time-interval of 2 weeks between replicates. Failure of individual cultures was accommodated by the setting up of a duplicate diallel on each occasion. A randomised block design was employed throughout the experiment which was carried out in an incubator maintained at $25^{\circ} \mathrm{C}$. Double pair matings were used to make up all 64 families of this diallel cross. Although the sternopleural chaetæ numbers of Io females and Io males were scored from each culture, only the female results will be given here in order to avoid complications which might arise from the male sex chromosome.

\section{ANALYSIS OF VARIANCE}

An analysis of variance essentially similar to the one devised and described by Hayman (1954) was carried out on the results obtained. This showed beyond doubt the presence amongst these 8 lines of significant genetic variation ( $a$ item of table I) and also of dominance at some of the loci controlling sternopleural chaetæ number ( $b$ item). Furthermore the non-significant $(c)$ and $(d)$ items indicated that any maternal effects present were negligible. It is possible to subdivide the $(b)$ item into three parts $\left(b_{1}\right),\left(b_{2}\right)$ and $\left(b_{3}\right)$ measuring respectively the overall difference between parents and progeny, i.e., dominance, the consistency of the dominance effects over the lines and finally any specific differences which might occur between parental and progeny means. Neither the $\left(b_{1}\right)$ nor the $\left(b_{2}\right)$ items proved to be significant, but there was evidence from the highly significant $\left(b_{3}\right)$ item that the mean chaetæ numbers of some of the progeny do differ from those of their parents. Each mean effect was tested against a pooled error mean square with I89 degrees of freedom after a Bartlett test had shown the six individual error variances $\mathrm{B} a$ to $\mathrm{B} d$ to be homogeneous $\left(\chi_{[5]}^{2}=10 \cdot 139, P=0 \cdot 10-0 \cdot 05\right)$. Despite the significance of the main 
TABLE I

Analysis of variance of the diallel table

\begin{tabular}{|c|c|c|c|c|c|}
\hline Item & D.F. & S.S. & M.S. & V.R. & $\mathbf{P}$ \\
\hline $\begin{array}{l}a \\
b_{1} \\
b_{2} \\
b_{3}\end{array}$ & $\begin{array}{r}7 \\
1 \\
7 \\
20\end{array}$ & $\begin{array}{r}25,138 \cdot 0000 \\
57 \cdot 5714 \\
313 \cdot 0000 \\
1,342 \cdot 4286\end{array}$ & $\begin{array}{r}359 \mathrm{r} \cdot 1428 \\
57 \cdot 5714 \\
44 \cdot 7 \mathrm{I} 43 \\
67 \cdot 1214\end{array}$ & $\begin{array}{r}\text { I } 5.0348 \\
1.8442 \\
1.4323 \\
2.1502\end{array}$ & $\begin{array}{l}<0.001 \\
0 \cdot 2-0 \cdot 1 \\
>0 \cdot 2 \\
0 \cdot 01-0.001\end{array}$ \\
\hline$b$ & 28 & $\mathrm{I}, 713 \cdot 0000$ & $6 r \cdot 1786$ & I 9598 & $0.01-0.001$ \\
\hline $\begin{array}{l}c \\
d\end{array}$ & $\begin{array}{r}7 \\
21\end{array}$ & $\begin{array}{r}133 \cdot 6250 \\
80 \cdot 3750\end{array}$ & $\begin{array}{r}19.0893 \\
3.8274\end{array}$ & $\ldots$ & $\begin{array}{l}\ldots \\
\ldots\end{array}$ \\
\hline$t$ & 63 & $27,065 \cdot 0000$ & & & \\
\hline $\begin{array}{l}\mathrm{B} \\
\mathrm{B} a \\
\mathrm{~B} b_{1} \\
\mathrm{~B} b_{2} \\
\mathrm{~B} b_{3} \\
\mathrm{~B} c \\
\mathrm{~B} d\end{array}$ & $\begin{array}{r}3 \\
21 \\
3 \\
21 \\
60 \\
21 \\
63\end{array}$ & $\begin{array}{r}621 \cdot 9600 \\
509 \cdot 0000 \\
35 \cdot 6384 \\
249 \cdot 0000 \\
2149 \cdot 3616 \\
636 \cdot 8750 \\
2320 \cdot 1250\end{array}$ & $\begin{array}{r}207 \cdot 3200 \\
24 \cdot 238 \mathrm{I} \\
\mathrm{I} \mathrm{I} \cdot 8795 \\
\mathrm{I} \mathrm{I} \cdot 857 \mathrm{I} \\
35 \cdot 8227 \\
30 \cdot 3274 \\
36 \cdot 8274\end{array}$ & $6.64 \times 3$ & $<0.001$ \\
\hline $\mathrm{B} t$ & 189 & $5900 \cdot 0000$ & $3 r \cdot 2 r 69$ & & \\
\hline Total & 255 & $33,586 \cdot 9600$ & & & \\
\hline
\end{tabular}

block effect there were no major alterations in the relative performance of the lines over the different blocks. Had there been such alterations the $\mathrm{B} a$ and $\mathrm{B} b$ items would have been significant by comparison with the $\mathrm{B} c$ and $\mathrm{B} d$ items.

\section{THE ANALYTIC AND SYNTHETIC PARAMETERS}

Amongst the 64 families comprising the present diallel cross there are 27 distinct genotypes. The variation between these genotypes can be described in terms of 26 genetic parameters each with a single degree of freedom. Estimates of these parameters are derived from the different genotype means, weighted according to the number of families which contribute to them, by a series of orthogonal comparisons (see tables 2 and 3 ). Their calculation follows the procedure devised by Cooke and Mather ( 1962 ) except that all the divisors have been increased to take into account the greater size of this experiment.

A second set of parameters was computed directly from the family means by unweighted least squares (see Mather, 1949), so that the total genetic variation amongst the parental lines could be partitioned 
TABLE 2

Mean chaete number averaged over blocks and genotypes, and number of families contributing to each genotype mean

\begin{tabular}{|c|c|c|c|c|c|c|c|c|c|}
\hline II & \multicolumn{3}{|c|}{ BB } & \multicolumn{3}{|c|}{ BS } & \multicolumn{3}{|c|}{ SS } \\
\hline & BB & BS & SS & BB & BS & SS & BB & BS & SS \\
\hline \multirow[t]{2}{*}{ BB } & $22 \cdot 0250$ & $21 \cdot 6125$ & $19 \cdot 6250$ & $21 \cdot 0875$ & $20 \cdot 1563$ & 19.1125 & 19.9250 & $19.475^{\circ}$ & 18.4500 \\
\hline & 4 & 8 & 4 & 8 & 16 & 8 & 4 & 8 & 4 \\
\hline \multirow{2}{*}{ BS } & $22 \cdot 2000$ & $21 \cdot 0250$ & $19 \cdot 6625$ & $21 \cdot 1375$ & $19 \cdot 8969$ & 18.9250 & $20 \cdot 2375$ & $19 \cdot 0875$ & $18.275^{\circ}$ \\
\hline & 8 & 16 & 8 & 16 & 32 & 16 & 8 & 16 & 8 \\
\hline \multirow[t]{2}{*}{ SS } & $22 \cdot 0250$ & $21 \cdot 1500$ & $19 \cdot 675^{\circ}$ & $20 \cdot 9625$ & 20.0313 & $19.175^{\circ}$ & $19.775^{\circ}$ & $19 \cdot 1375$ & $18 \cdot 1000$ \\
\hline & 4 & 8 & 4 & 8 & 16 & 8 & 4 & 8 & 4 \\
\hline
\end{tabular}

\section{TABLE 3}

Estimates of the parameters obtained for each chromosome separately (a) from genotype means and (b) by unweighted least squares from family means

\begin{tabular}{|c|c|c|}
\hline Parameter & (a) & (b)--averaged over blocks \\
\hline 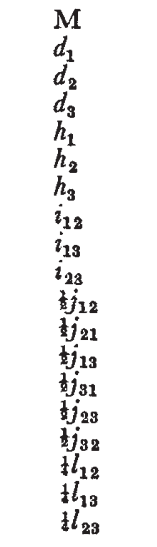 & $\begin{array}{r}\ldots \\
0.0789 \\
0.9430 \\
1.0398 \\
-0.1086 \\
-0.0836 \\
0.0570 \\
-0.0188 \\
0.0141 \\
0.1719 \\
-0.0445 \\
-0.0297 \\
0.0430 \\
0.0758 \\
0.0703 \\
-0.0164 \\
-0.0133 \\
-0.0719 \\
-0.0601\end{array}$ & $\begin{array}{c}20.0876 \pm 0.0721 * * * \\
0.0836 \pm 0.0510 \\
0.9328 \pm 0.0510^{* * *} \\
1.0399 \pm 0.0510^{* * *} \\
-0.0961 \pm 0.0721 \\
-0.0844 \pm 0.0721 \\
0.0578 \pm 0.0721 \\
-0.0188 \pm 0.0676 \\
0.0141 \pm 0.0676 \\
0.1719 \pm 0.0676^{*} \\
-0.0445 \pm 0.0478 \\
-0.0297 \pm 0.0478 \\
0.0430 \pm 0.0478 \\
0.0758 \pm 0.0478 \\
0.0703 \pm 0.0478 \\
-0.0164 \pm 0.0478 \\
-0.0133 \pm 0.0338 \\
-0.0719 \pm 0.0338 * \\
-0.0601 \pm 0.0338\end{array}$ \\
\hline
\end{tabular}

into either additive or dominance or first-order interaction effects. After fitting the parameters to the data in the sequence:-

(i) mid-parent and additive parameters,

(ii) mid-parent, additive and dominance parameters,

(iii) mid-parent, additive and first-order interaction parameters, 
it is possible to test analytically the goodness of fit of these three models to the data and hence calculate the sum of squares attributable to each set of parameters in turn. Since these sums of squares were calculated by squaring and summing the deviations between the observed and expected values, there were a total of 256 degrees of freedom initially available for each analysis, of which $n$ were taken up in fitting $n$ parameters to the data. The remaining degrees of freedom can be assigned as follows: $192=3 \times 64$ to the within family between

TABLE 4

Degrees of freedom and mean squares for the analysis of the unweighted least squares estimates of the parameters; (i) mid-parent and additive parameters; (ii) mid-parent, additive and dominance parameters and (iii) mid-parent, additive and interaction parameters where $\mathrm{g}=$ number of genotypes; $\mathrm{n}=$ number of parameters fitted; $\mathrm{a}=$ number of families in the diallel cross and $\mathrm{b}=$ number of blocks

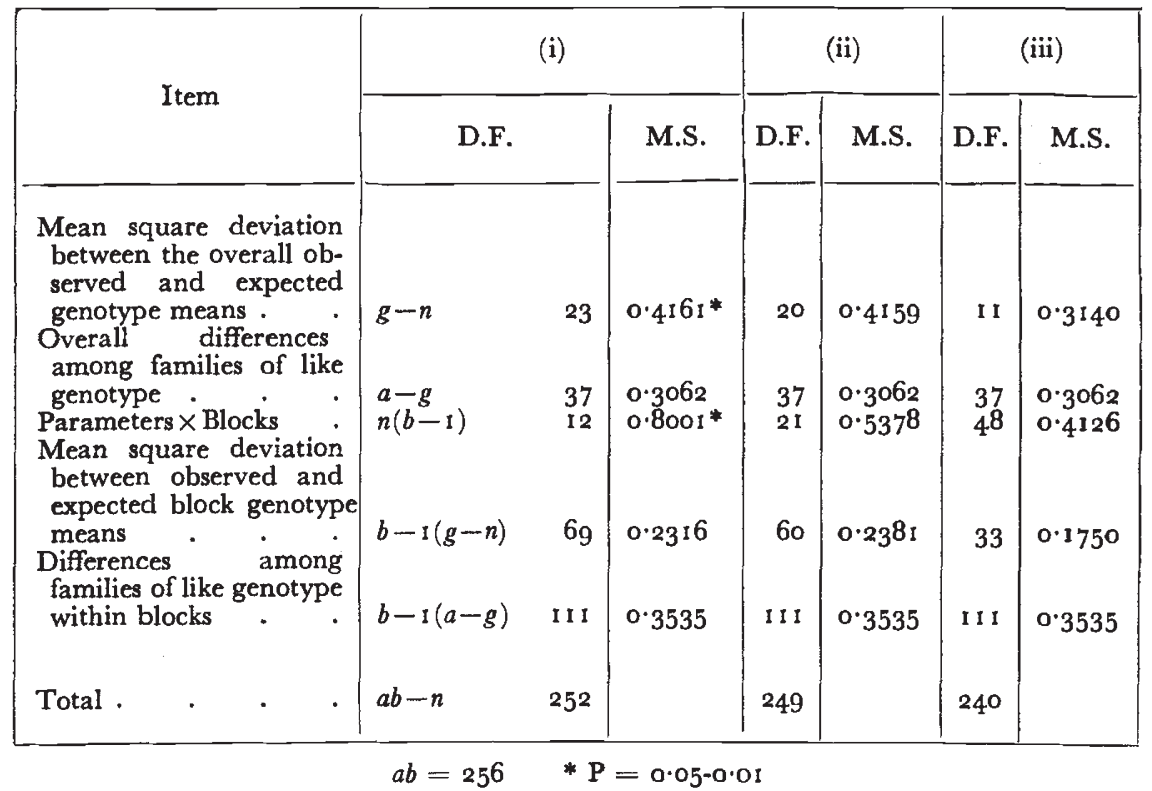

blocks item leaving $256-n-\mathrm{I} 92=64-n$ to the remaining variation or residual heterogeneity. Of these $64-n$ d.f., $27-n$ can be allocated to the deviation from expectation of the overall genotype means, whilst the remaining 37 represent differences between families within the same genotype again summed over blocks. This last item will be constant throughout all three analyses. The within family between blocks item can be subdivided into three parts, one measuring the components $\times$ blocks interaction with $n \times 3$ d.f., another the deviation between the observed and expected genotype means within blocks which has $3(27-n)$ d.f. and finally an item having $3 \times 37=$ I I I d.f. which can be ascribed to differences between families of similar genotypes within blocks. This latter, which is also constant throughout, is calculated by accumulating the differences between families of like 
genotype within blocks and then subtracting from it the sum of squares and degrees of freedom obtained for the variation due to these families when pooled over blocks. The goodness of fit of any one of these models can be determined by comparing the mean square deviation of the overall genotype mean with the corresponding within blocks item. Whilst this comparison proves to be significant at the 5 per cent. level of probability when testing the fit of the mid-parent plus additivity model, it is not significant either for the dominance or first-order interaction models (table 4). This would seem to suggest that although neither dominance nor first-order interactions by itself can account for a significant amount of the genetic variation, their combined effects

TABLE 5

Sum of squares due to (i) dominance and (ii) first-order interactions

\begin{tabular}{|c|c|c|c|c|c|}
\hline \multicolumn{6}{|c|}{ (i) } \\
\hline Item & D.F. & S.S. & M.S. & V.R. & $P$ \\
\hline $\begin{array}{l}\text { Main effect } \\
\text { Block heterogeneity }\end{array}$ & $\begin{array}{l}3 \\
9\end{array}$ & $\begin{array}{l}\mathrm{I} \cdot 2532 \\
\mathrm{I} \cdot 6928\end{array}$ & $\begin{array}{l}0.4177 \\
0.1881\end{array}$ & $2 \cdot 22$ & $0.2-0 . I$ \\
\hline \multicolumn{6}{|c|}{ (ii) } \\
\hline $\begin{array}{l}\text { Main effect } \\
\text { Block heterogeneity }\end{array}$ & $\begin{array}{l}12 \\
3^{6}\end{array}$ & $\begin{array}{r}6 \cdot 1168 \\
10 \cdot 2042\end{array}$ & $\begin{array}{l}0.5097 \\
0.2834\end{array}$ & $1 \cdot 79$ & 0.1 \\
\hline
\end{tabular}

may possibly make a significant contribution to the total variation present amongst these lines. To confirm this the relevant sums of squares were calculated by assessing the improvement brought about as a result of fitting successively more parameters to the data. Subtraction of the deviation sum of squares between the expected and observed overall genotype means of analysis (ii) from the corresponding item in analysis (i) of table 4 will provide a direct measure of the amount of genetic variation ascribable to dominance, whilst an estimate of its error can be obtained by taking the within block deviations of analysis (ii) away from analysis (i). Likewise subtraction of these items in analysis (iii) from those of analysis (i) will yield estimates of the variation due to first-order interactions and its error (table 5). Although in fact neither dominance nor first-order interactions alone prove to be significant, nevertheless their joint effect is bordering on significance at the 5 per cent. level of probability. This leads us to suspect that perhaps the estimates of one or both these sets of parameters are considerably below their true value. Evidence will be presented at a later stage from the $\mathrm{Wr} / \mathrm{Vr}$ graphs substantiating the view that it is the level of dominance rather than the level of first-order interactions which has been underestimated by the chromosomal parameters. 
Since the exact chromosomal constitution of all 8 lines used in this experiment is known, it has been possible to calculate the values of the genetic parameters for each chromosome separately. In general this cannot be done, the more usual procedure being to estimate the additive and dominance effects summed over all the genes concerned with a particular character, viz., $\mathrm{D}$ and $\mathrm{H}$. These components can also be computed for this experiment, again by unweighted least squares, from the following statistics: mean variance of arrays $\left(\mathrm{V}_{I L I}\right)$; variance of array means around the grand mean $\left(\mathrm{V}_{O L I}\right)$; covariance of offspring onto parents $\left(\mathrm{W}_{O L O I}\right)$ and the variance of parental means around the mid-parent $\left(\mathrm{V}_{\text {OLo }}\right)$. Reciprocal differences within blocks provide direct estimates of the environmental variation $\left(\mathrm{E}_{2}\right)$. The expectation for each of these statistics is given by Jinks (1954), but

TABLE 6

Comparison of analytic and synthetic estimates of the components and dominance levels

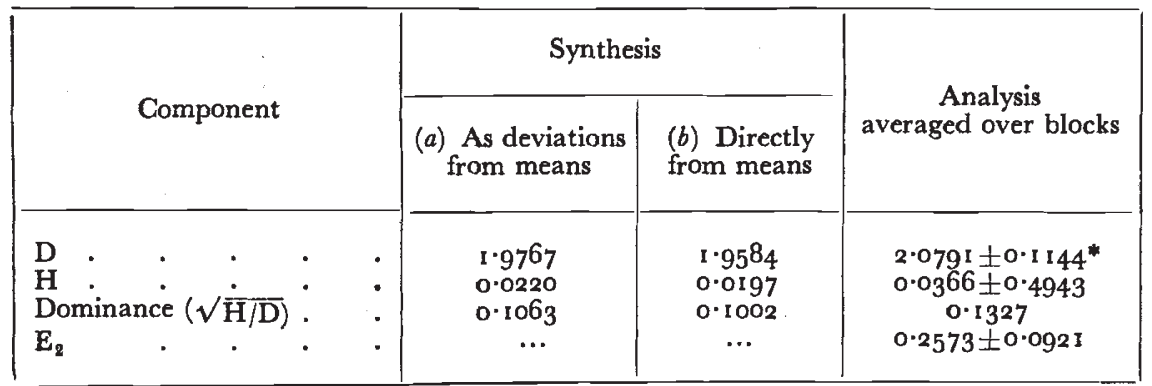

$* \mathrm{P}=<0 \cdot \mathrm{Or}$

since the structure of the present experiment is such that there are equal numbers of genes increasing or decreasing sternopleural chaetæ number (i.e. $u=v=\frac{1}{2}$ ), they can be simplified to become

$$
\begin{aligned}
\mathrm{V}_{\text {OLO }} & =\mathrm{D}+\quad \mathrm{E}_{2} \\
\mathrm{~V}_{\text {ILI }} & =\frac{1}{4} \mathrm{D}+\frac{1}{4} \mathrm{H}+\mathrm{E}_{2} \\
\mathrm{~V}_{\text {OLI }} & =\frac{1}{4} \mathrm{D}+\quad \frac{1}{8} \mathrm{E}_{2} \\
\mathrm{~W}_{\text {OLOI }} & =\frac{1}{2} \mathrm{D}+\quad \frac{1}{8} \mathrm{E}_{2}
\end{aligned}
$$

where D takes the special value of $\Sigma\left(d^{2}\right)$ and similarly $\mathrm{H}$ is equal to $\Sigma\left(h^{2}\right)$. Standard errors can be attached to these " analytic" estimates (see Cooke and Mather, I 1962) as there are more statistics available than components to be calculated (table 6). Analysis of these components shows that a model based on them accounts for a significant amount of the variation which occurs amongst the lines, thus demonstrating once more the almost total absence of non-allelic interactions (table 7).

Having derived values of the components for each chromosome separately as well as an independent overall estimate, it is of interest to compare them especially as any differences between them will, in 
this experiment, reflect mainly the presence of gene interactions. By squaring and summing the chromosomal parameters, "synthetic" estimates of the components are obtained which are equivalent to and hence comparable with the "analytic" estimates. Reference to table 6 , where the values for both sets of estimates are given, clearly indicates that the "synthetic" components are remarkably similar to their " analytic" counterparts, thereby confirming the virtual absence of any interactions between the different genes controlling the expression of sternopleural chaetæ number in these flies.

As has been mentioned earlier the calculation of the synthesised parameters follows closely the method devised by Cooke and Mather.

TABLE 7

Analysis of the analytic components $D, H$ and $E_{2}$ estimated from statistics obtained by diallel analysis

\begin{tabular}{|c|c|c|c|c|c|}
\hline Item & D.F. & S.S. & M.S. & V.R. & $\mathbf{P}$ \\
\hline $\begin{array}{l}\text { Components } \times \text { Blocks } \\
\text { Residual Heterogeneity } \\
\text { Block Heterogeneity }\end{array}$ & $\begin{array}{l}9 \\
2 \\
6\end{array}$ & $\begin{array}{l}0.5577 \\
0 \cdot 0760 \\
0 \cdot 1485\end{array}$ & $\begin{array}{l}0.0620 \\
0.0380 \\
0.024^{8}\end{array}$ & $\begin{array}{l}2 \cdot 5000 \\
1 \cdot 5323\end{array}$ & $\begin{array}{l}\text { N.S. } \\
\text { N.S. }\end{array}$ \\
\hline Total & 17 & 0.7822 & & & \\
\hline
\end{tabular}

Since they also obtained "analytic" estimates their results may be compared with those presented here. With few exceptions the values of the two sets of chromosomal parameters and, therefore, of the "synthetic" estimates are in broad agreement. The main difference between the two sets of results lies in the fact that the "analytic" estimates of Cooke and Mather's experiment are approximately 40 per cent. higher than their corresponding "synthetic" values. Such a difference is, however, to be expected in their experiment if only because their "analytic" estimates were calculated from the segregating $F_{2}$ and BIP generations, and not as they were here from the non-segregating $F_{1}$ generation. Strictly speaking, therefore, it is not possible to compare Cooke and Mather's "synthetic " and " analytic" estimates directly as the latter will have been inflated by the genetical variation released by recombination. Thus the divergences which occur between the two experiments on this particular issue reflect basically the differing techniques used and are not due to any more fundamental reason.

\section{PRODUCTION OF CORRELATED GENE DISTRIBUTIONS}

(i) Development of techniques

Before actually attempting to simulate the effects of correlated gene distributions on the experimental data, it is important to verify the 
suitability of the procedures to be used. For this purpose a 3-gene model was adopted since, with all the parental lines true-breeding, a whole chromosome can be equated to the genetic unit or gene. Association and dispersion imply that genes of similar or opposite effects respectively occur together with a greater frequency than is expected solely on the basis of random assortment. Assuming dominance to be both complete and reinforcing (i.e., $d_{a}=d_{b}=d_{c}=h_{a}=h_{b}=h_{c}$ $=\mathrm{I}), \mathrm{Wr} / \mathrm{Vr}$ graphs demonstrating the effects of association can be obtained by increasing the frequency of the extreme AABBCC and aabbcc arrays at the expense of the central AAbbcc and aaBBCC arrays, whereas the reverse process gives rise to a graph which displays the effects of dispersion (Jinks, I956). Variations in the dominance relationship such that it is assumed to be opposing $\left(d_{a}=d_{b}=d_{c}\right.$ $\left.=h_{a}=-h_{b}=-h_{c}=\mathrm{I}\right)$ or both incomplete and opposing $\left(d_{a}=d_{b}\right.$ $=d_{c}=2 h_{a}=-2 h_{b}=-2 h_{c}=\mathrm{I}$ ) appear to have no appreciable effect either on the type of graph or on the magnitude of the disturbance created. However, because of these alterations the order of array points along the graph is no longer the same as when reinforcing dominance is assumed to be present. Finally, and in order to ensure the maintenance of equal gene frequencies throughout, corresponding arrays were always increased or decreased in proportion to one another.

\section{(ii) Application to the experimental data}

The correctness of the techniques to be adopted having been established from a model, they could then be applied to the experimental results. The complete female data were therefore arranged according to their parental genotypes in a diallel table. Each of the 64 family squares in the table was subdivided into 4 cells. Allowances for the block differences referred to earlier were made by allotting the results of an individual cross from a particular block at random to one of the cells within the appropriate family square. This produced a diallel table in which effectively both rows and columns had been doubled up (table 8). A conventional diallel analysis was then carried out on the complete set of data using mean chaetæ numbers averaged over reciprocals and cells as the metric.

On the basis of the hair counts of the parental lines and the diallel analysis BSS and SBB were selected as the two most extreme complementary arrays, whilst SBS and BSB were chosen as the two central arrays. These two pairs of arrays were subsequently used in all adjustments of the diallel table required for the generation of correlated gene distributions. Depending upon the number of times any one of the selected pairs of arrays is repeated within a particular table, so it is possible, at least for association, to achieve a gradual increase in the level of gene correlation. At each level four sets of array covariances and variances--one from each cell of a family square--were calculated with means averaged over reciprocals from corresponding cells. The formation of these adjusted tables may be illustrated numerically with 


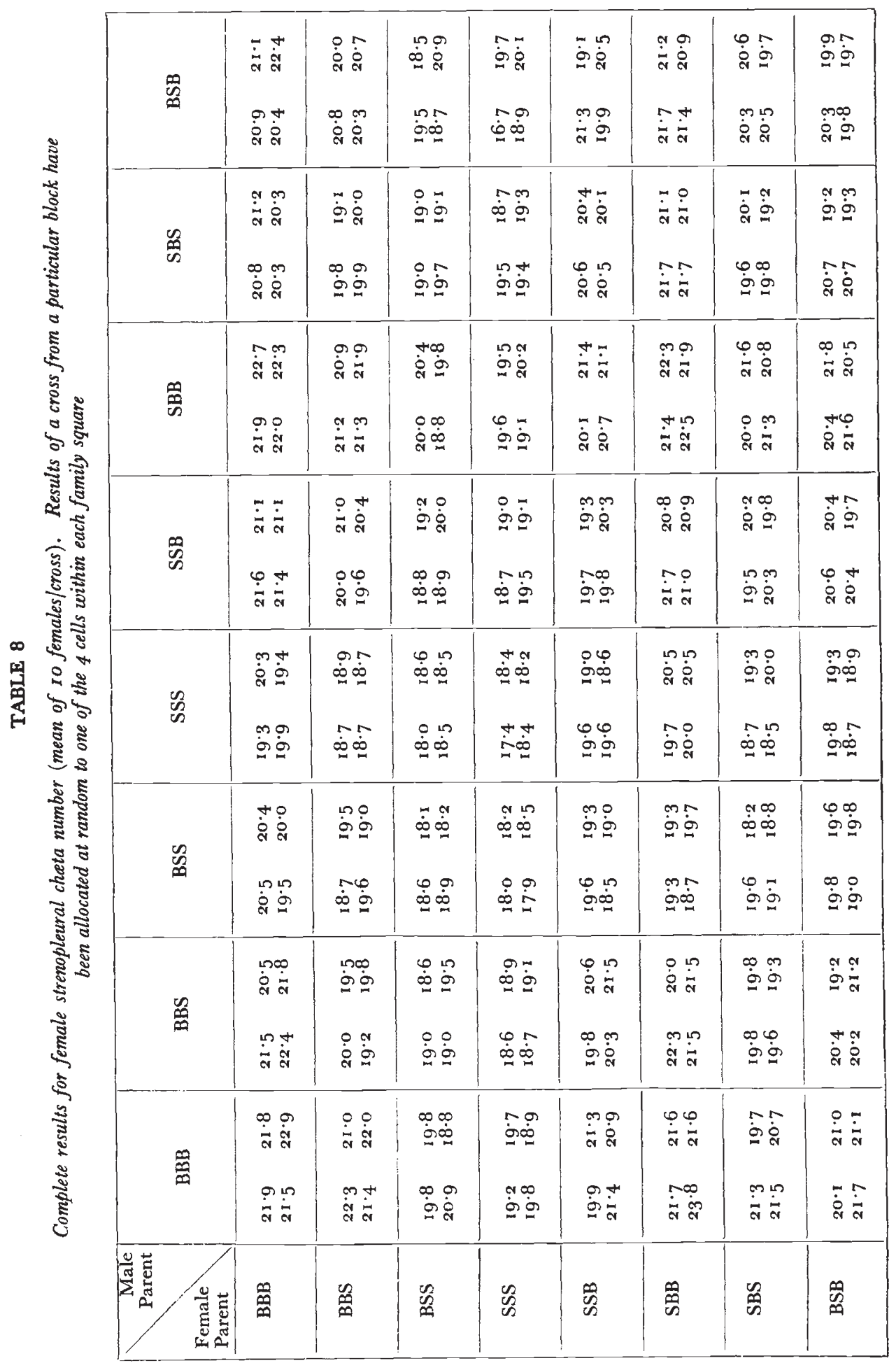




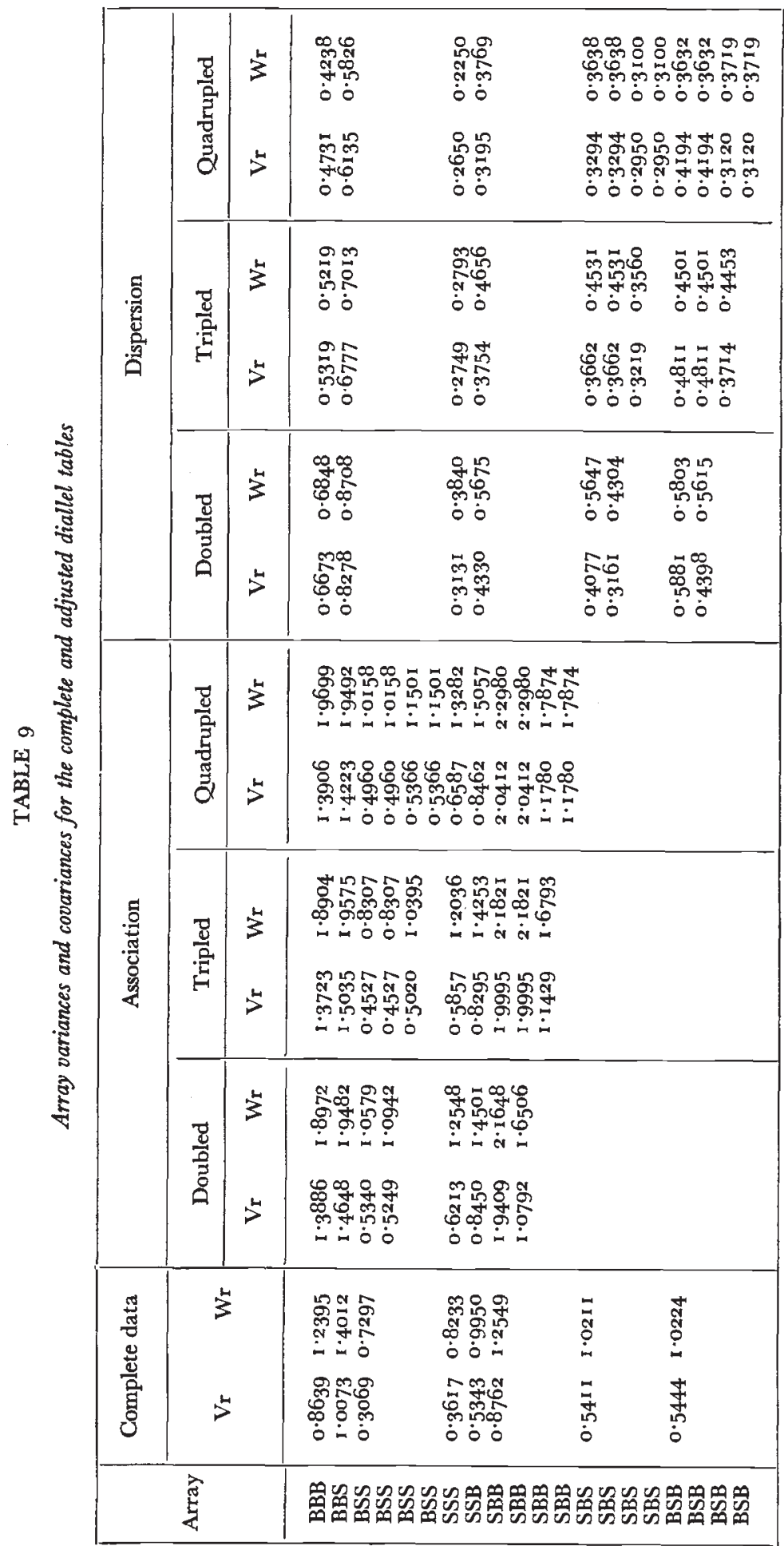


the means given in table 8 , even though these particular figures have not been averaged over reciprocals. Thus the BBB female array for the first of the four tables which results from doubling up the BSS and SBB arrays and omitting the SBS and BSB arrays would consist of the following figures:-2I.9, 2I.5, 20.5, 20.4, I9.3, 2I.6, $2 \mathrm{I} \cdot 9$ and $22 \cdot 7$;

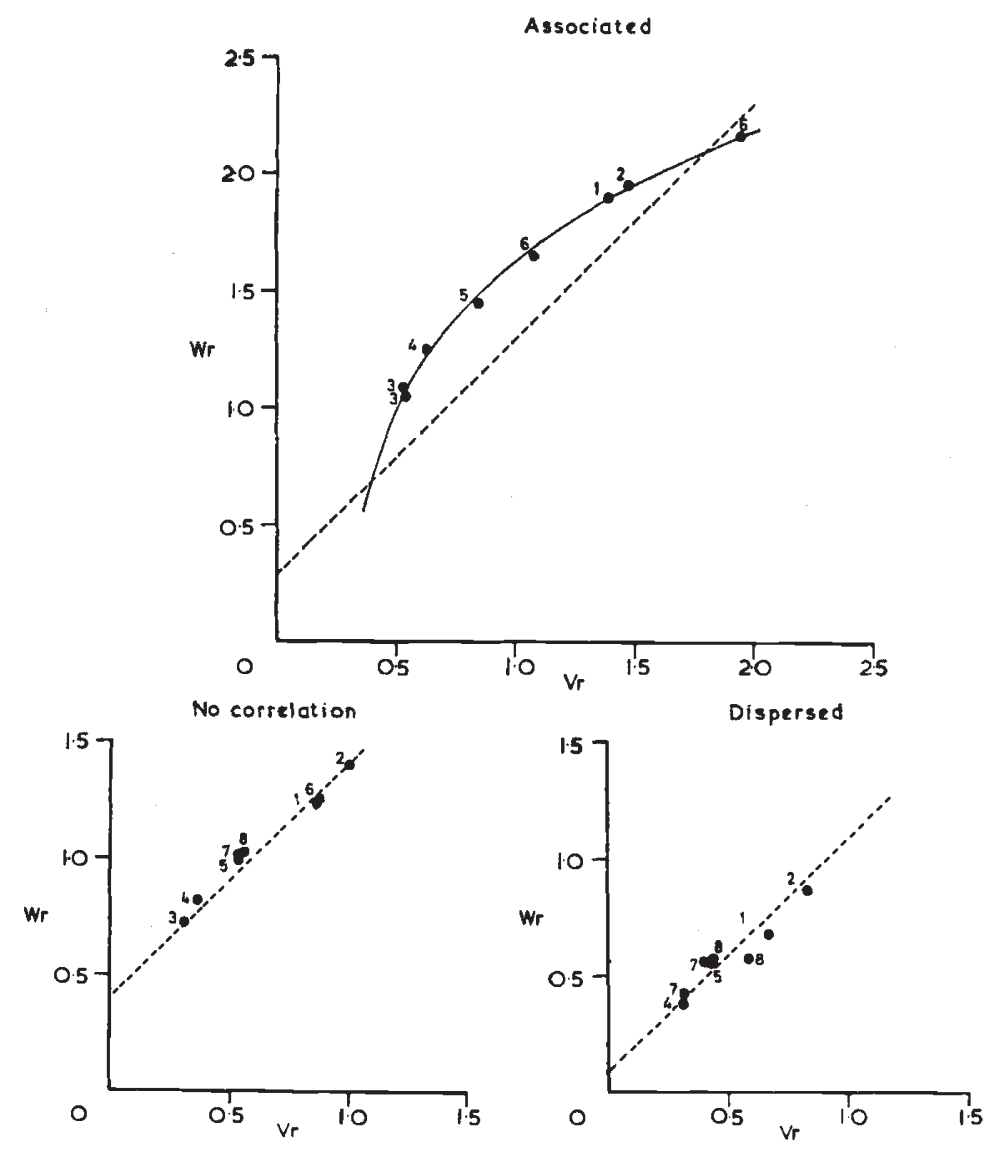

Fig, 1.-Wr/Vr graphs for the complete data and $66 \cdot 7$ per cent. association and dispersion obtained by doubling up and omitting appropriate arrays.

$$
\begin{array}{lllll}
\mathrm{Key}: & \mathrm{I}=\mathrm{BBB} & 2=\mathrm{BBS} & 3=\mathrm{BSS} & 4=\mathrm{SSS} \\
& 5=\mathrm{SSB} & 6=\mathrm{SBB} & 7=\mathrm{SBS} & 8=\mathrm{BSB}
\end{array}
$$

while for the fourth table it would contain $22 \cdot 9,2 \mathrm{I} \cdot 8,19 \cdot 5,20 \cdot 0,19 \cdot 4$, $2 \mathrm{I} \cdot \mathrm{I}, 22 \cdot 0$ and $22 \cdot 3$. When the selected arrays were only represented twice in a diallel table duplication of results could be entirely avoided by the use of means from different replicates of a given family. If, however, these arrays were present 3 or 4 times within a table then a certain amount of repetition was unavoidable. Again taking the BBB array as an example, tripling the two extreme arrays would give the following figures for the first table:-2I.9, $2 \mathrm{I} \cdot 5,20 \cdot 5,20^{\circ} 5,20 \cdot 4,19 \cdot 3$, $2 \mathrm{I} \cdot 6,2 \mathrm{I} \cdot 9,2 \mathrm{I} \cdot 9$ and $22 \cdot 7$ 
In diallel analysis the first indication that one of the initial assumptions is not valid is usually a significant departure between the observed and expected slope of the regression line drawn to the $\mathrm{Wr} / \mathrm{Vr}$ graph. Clearly therefore it is necessary to establish whether in practice the graph is sufficiently sensitive to permit detection of non-random gene
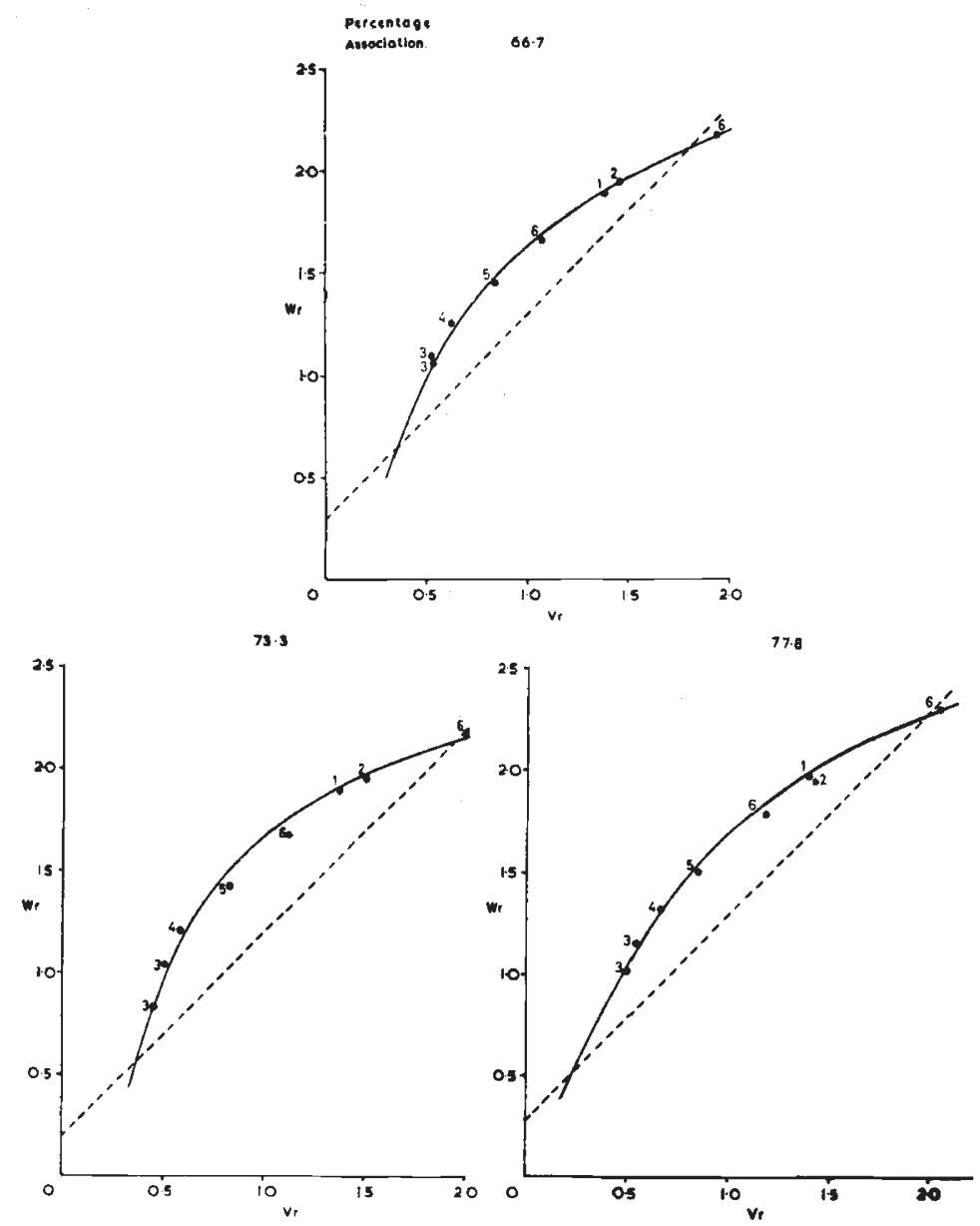

Fig. 2.-Effects of different levels of association on the $\mathrm{Wr} / \mathrm{Vr}$ graph-actual data. Key as for fig. 1 .

distributions when they are present. Accordingly a series of $\mathrm{Wr} / \mathrm{Vr}$ graphs were constructed from the array covariances and variances of both the adjusted and unadjusted diallel tables (table 9). These show that associated distributions produce a distinct upward curvature to the graph, but the downward curvature expected when dispersed combinations are in excess is not discernable (fig. I). This is because of a tendency for dispersion to give rise to a graph with an apparently random scatter of points when the actual data are used.

As previously noted increasing levels of correlation can be generated 
for associated distributions. Each successive increase leads to a greater range of array covariances and variances and this in turn widens the spread of the graph (fig. 2). A comparison between the effects on both the model and the data of identical increases in the level of association can be made from figs. 2 and 3. The striking similarities between the two sets of graphs serve only to confirm the suitability
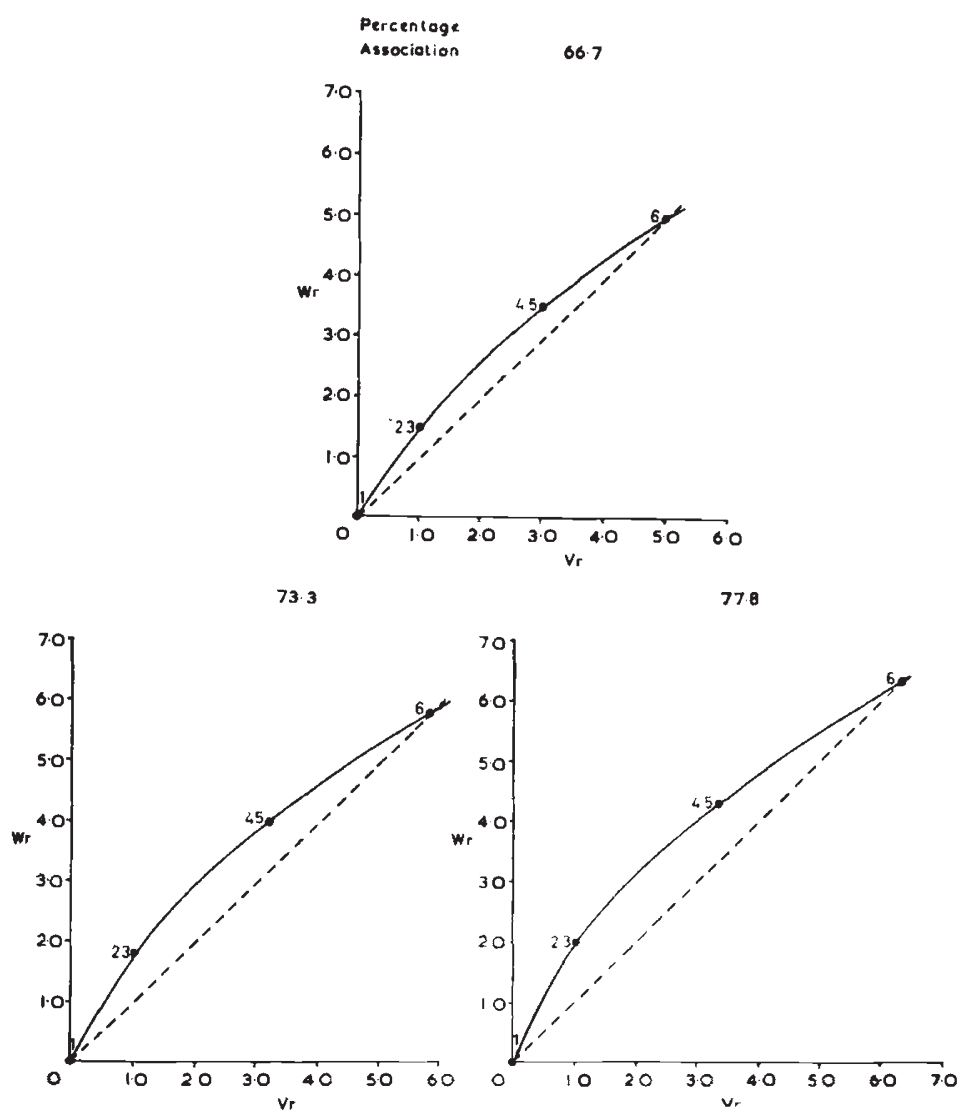

FrG. 3.-Effects of different levels of association on the $\mathrm{Wr} / \mathrm{Vr}$ graph assuming complete and reinforcing dominance- 3 gene model.

$$
\begin{aligned}
& \text { Key: } \mathrm{I}=\mathrm{AABBCC} \\
& 4=\mathrm{aaBBcc} \\
& \begin{array}{l}
2=\mathrm{AABBcc} \\
5=\mathrm{a} \text { abCC }
\end{array} \\
& 3=\mathrm{AAbbCC} \\
& 6=a a b b c c
\end{aligned}
$$

of the methods developed as a means of assessing the gross effects of correlated gene distributions.

In order to examine these effects in greater detail, however, it is necessary to apply statistical analysis to the data and the model before and after adjustment. This is achieved by fitting to the $\mathrm{Wr} / \mathrm{Vr}$ graphs multiple regression curves of the form $Y=\bar{y}+b_{1}\left(x_{1}-\bar{x}_{1}\right)+b_{2}\left(x_{2}-\bar{x}_{2}\right)$, where $x_{1}=\mathrm{Vr}, x_{2}=\mathrm{Vr}^{2}$ and $y=\mathrm{Wr}$. Comparing first the results of the analyses of these regression curves for the adjusted diallel tables 
GENE DISTRIBUTIONS IN DIALLELS

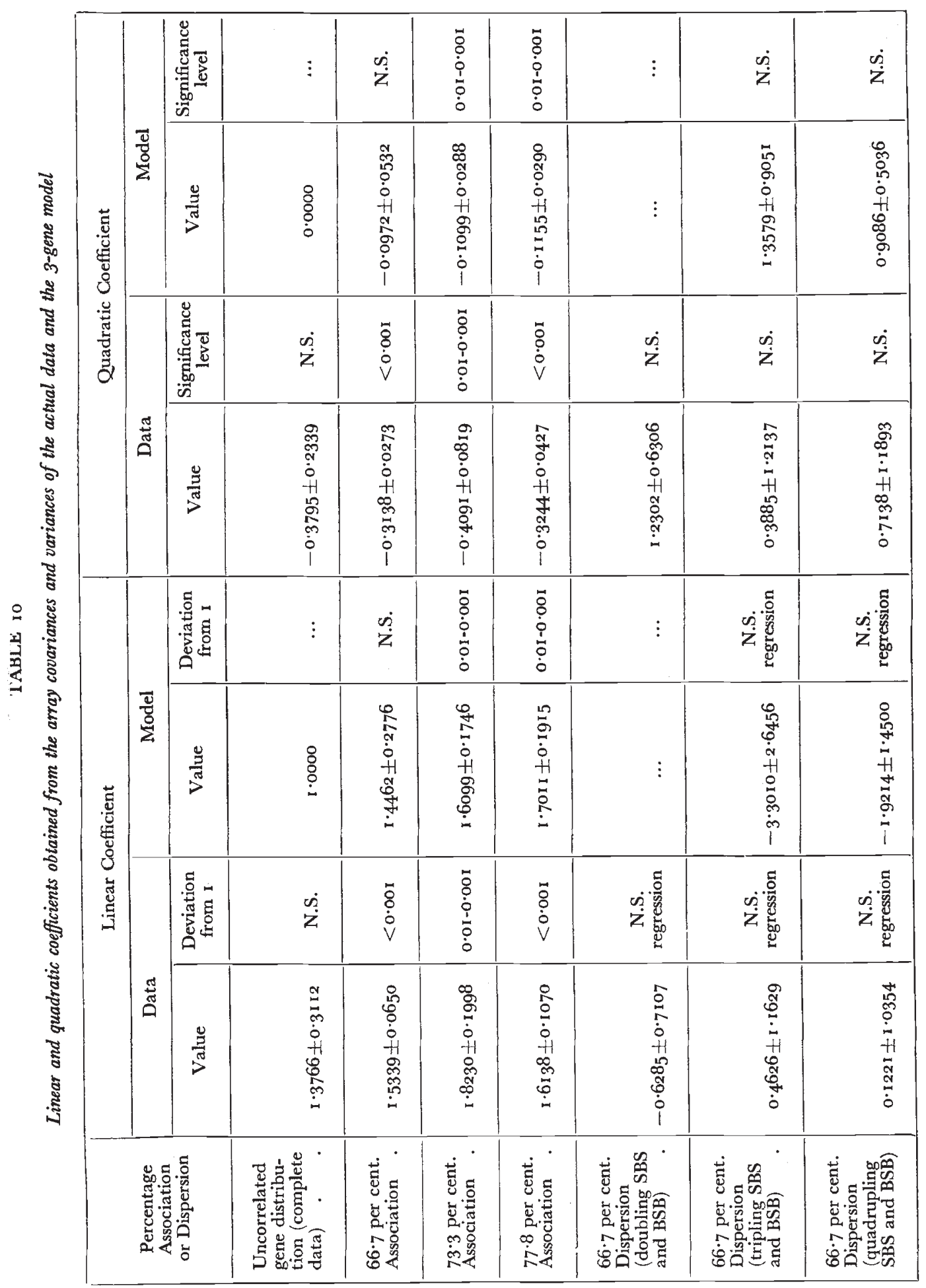


of both model and experiment (table Io), it is evident that for association they confirm the impression gained from the respective $\mathrm{Wr} / \mathrm{Vr}$ graphs (see figs. 2 and 3), namely that it is possible to predict with accuracy from the model situation the nature and magnitude of the disturbance created. Turning to dispersion here also there are close similarities between the two situations which may appear somewhat surprising in view of the fact that the graphs obtained from the experimental results give no definite evidence of curvature. Thus, although the graphs for the model suggest that a curve might possibly be drawn to the array points, the analyses on the other hand show that there is neither a significant curvature nor even a significant regression line. This is fully borne out by the corresponding analyses on the actual data. If, however, it is remembered that in generating an excess of dispersed gene combinations the extreme arrays are deliberately excluded, this result is, perhaps, not entirely unexpected, since the remaining arrays will contain approximately the same proportion of dominant and recessive genes and hence will lie at similar places on the $\mathrm{Wr} / \mathrm{Vr}$ graph. Whereas exclusion of the extreme arrays will lead to a reduction in the level of genetic variation, it in no way reduces the amount of environmental variation present. Under such circumstances the array points will tend to be scattered at random throughout the central region of the $\mathrm{Wr} / \mathrm{Vr}$ graph instead of forming a distinct curve as they do with associated gene distributions.

Considering the analyses of the unadjusted diallel tables, the model shows no departure from linearity whatsoever. Indeed had it been otherwise serious doubts would have been cast upon the validity of the analysis. Likewise the regression line of the graph for the experimental results neither departs significantly from its expected slope of I nor gives any indication of curvature. This conformity to expectation of the $\mathrm{Wr} / \mathrm{Vr}$ graph for the experimental data returns us once more to the question of gene interactions. From the evidence presented here the conclusion seems to be that only an insignificant portion of the total genetic variation can be reliably attributed to non-allelic interactions. Admittedly the values for two of the first-order interaction parameters given in table 3 are significant and, moreover, it is also possible that their effects could easily be swamped in the diallel analysis by the insignificant effects which the majority of these components possess. But even so both components are only doubtfully significant at the 5 per cent. level when tested against their own errors.

It is on the issue of gene interactions that further differences arise between this experiment and the one referred to earlier conducted by Cooke and Mather. More specifically, certain of their first and second-order interactions proved to be significant. Furthermore, since an orthogonal set of parents was used in both experiments, arrays with complementary genotypes should occupy mirror-image positions along the $\mathrm{Wr} / \mathrm{Vr}$ graph, always providing that complicated gene interactions are absent. Whilst such an interrelationship between arrays exists in 
this experiment, it is lacking in Cooke and Mather's results. Differences of this type cannot be readily explained, but it is conceivable that they could be due to differences in the sampling procedures used in the two experiments.

Two other incidental effects of correlated gene distributions on the $\mathrm{Wr} / \mathrm{Vr}$ graph emerged during the course of this analysis. In the first place, such distributions have little if any effect on the order of array points along the graph. Estimates of the proportion of dominant to recessive genes which each parent contained would therefore remain virtually unaffected by the degree of association or dispersion. Secondly, correlated gene distributions do affect the apparent level of dominance: association results in its underestimation and dispersion in its overestimation.

\section{(iii) Measuring the effects of correlated distributions}

Whilst it is of obvious importance to be able to demonstrate the effects of correlated gene distributions, it is not sufficient to do this without relating the effects produced to the level of correlation attained. It is necessary therefore to develop metrics which will afford some means of measuring the non-randomness of a particular distribution. Two such metrics have been devised. The first depends upon the fact that with completely random gene distributions, half the gene combinations are associated and half dispersed. Representing increasing genes by $u$ and decreasing genes by $v$ and using two genes $a$ and $b$, this can be symbolised algebraically as

$$
u_{a} u_{b}+v_{a} v_{b}=u_{a} v_{b}+v_{a} u_{b}=\frac{1}{2} \text { when } u=v \text {. }
$$

In deriving the first metric we note that the product of associated $x$ dispersed combinations has a maximum value of $\frac{1}{4}$ when there is no correlation between the genes. The higher the correlation, the greater is the departure of this product from its maximum value. Hence

$$
\left\{\mathrm{I}-\left[4\left(u_{a} u_{b}+v_{a} v_{b}\right)\left(u_{a} v_{b}+v_{a} u_{b}\right)\right]\right\} \times 100
$$

will provide a measure of the non-randomness of a given distribution.

A second and equally valid estimate of the level of correlation present is obtained by expressing the number of associated and dispersed gene combinations as a percentage of the total possible combinations.

Either of these metrics will yield the requisite information, but for the sake of comparison the results of both are given in table II. These results point to a further important contrast between the two types of correlated gene distributions, namely that, whereas for association a range of correlations from 50-100 per cent. can occur, with dispersion the maximum correlation that can ever be achieved is 66.7 per cent. Such a restriction could conceivably play a subsidiary role in accounting 


\section{TABLE II}

Levels of association and dispersion produced by the procedures adopted in this experiment

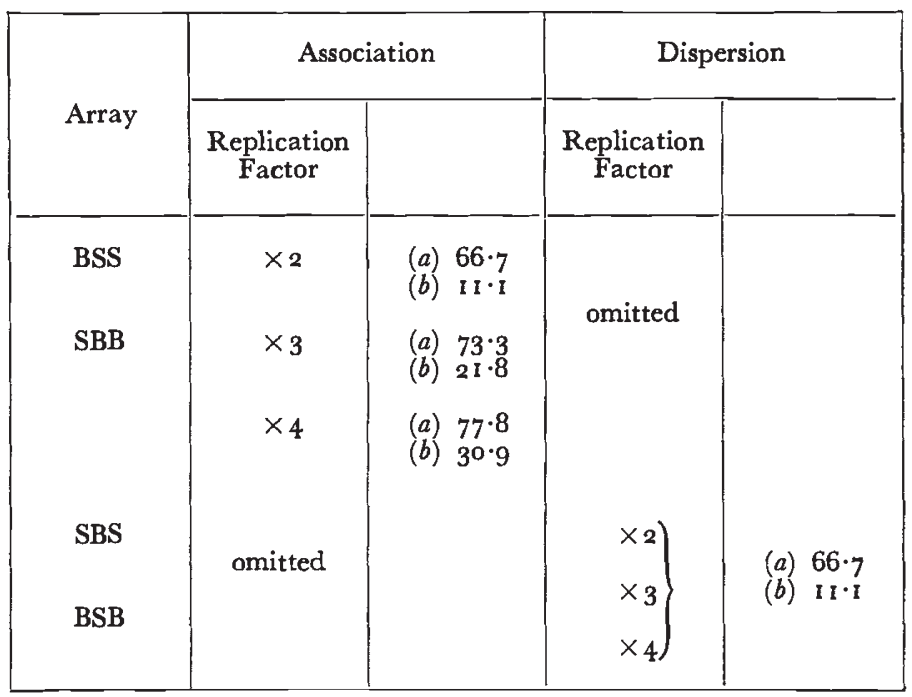

$(a)=$ percentage associated or dispersed gene combinations.

$(b)=$ percentage distortion created by the various treatments imposed.

for the inability of the $\mathrm{Wr} / \mathrm{Vr}$ graph to detect disturbances caused by dispersion.

\section{DISCUSSION}

Nowhere throughout these experiments, nor indeed in those of Cooke and Mather, has it been possible to demonstrate conclusively by the use of chromosome assay techniques that dominance exists amongst the genes determining sternopleural chaetæ number. Yet the evidence from the $\mathrm{Wr} / \mathrm{Vr}$ graphs suggests that a measurable level of dominance must be present because there is a clear separation of array points along the graph. This deduction follows from the fact that when dominance is absent all arrays are expected to have, within the limits of experimental error, the same covariance and variance and hence can give no regression of $\mathrm{Wr}$ on Vr. Providing some of the genes concerned with the character under investigation exhibit dominance, no matter how small, the spread of points along the graph is determined jointly by the dominance level and the number of gene differences between the two extreme parents. This appears to be the situation obtaining here since the significance of the $(b)$ item in the analysis of variance given in table I and also the suggestion from the analyses of the chromosomal parameters that if allowances for dominance and first-order interactions are made there is a measurable improvement in the goodness of fit, indicate that there is a certain minimum level of dominance with possibly gene interactions present 
at some of the loci controlling sternopleural chaetæ number. To what then are the apparently insignificant levels of dominance attributable? At this stage it is not possible to give precise reasons as to why the chromosome estimates should fail to detect dominance. It may be that before dominance can be detected by assay techniques the individual unit whose effect it is desired to measure will have to be considerably smaller than the chromosome itself. This is not to say that splitting the chromosomes up into smaller units will necessarily allow overall dominance to be detected, but it should at least enable those regions of particular chromosomes which exhibit the most marked dominance to be located. Such an assay would naturally entail the use of marker genes so that each segment of the chromosome could be accurately defined (see Breese and Mather, 1957). What is apparent from the present investigation, however, is that the $\mathrm{Wr} / \mathrm{Vr}$ graph is able, again for reasons which are not yet clearly understood, to detect overall dominance even though other methods of detection have failed. Presumably the $\mathrm{Wr} / \mathrm{Vr}$ graph can take account of all these considerations simultaneously, whilst the other methods of estimation cannot. Provided therefore that no gene interactions occur, the $\mathrm{Wr} / \mathrm{Vr}$ graph and Hayman's analysis of variance would appear to yield more sensitive tests for dominance than estimates of the appropriate parameters.

Further evidence for the presence of dominance comes from the noticeable similarities already mentioned between the 3-gene model developed for this experiment and the actual experimental results. Included in the model are the twin assumptions of complete dominance and total absence of non-allelic interactions. The very fact that it is possible to predict with accuracy from the regression analyses and $\mathrm{Wr} / \mathrm{Vr}$ graphs of this model the type and magnitude of the disturbance to be expected in practice when correlated gene distributions occur, suggests strongly that the level of dominance calculated from the parameter estimates is spuriously low.

From these results it is apparent that the main effects of correlated gene distributions are clearly recognisable and, moreover, that association on the one hand can be distinguished from dispersion on the other. One important question remains unanswered. Is it possible to discriminate between the effects of correlated gene distributions and those of gene interactions? This is an important question because the two mimic one another to a certain extent in the nature of the disturbance which each can create on the $\mathrm{Wr} / \mathrm{Vr}$ graph. To this end an investigation is at present in progress which it is hoped will enable tests to be devised permitting such a distinction to be drawn. Should these tests become feasible they would be of the utmost practical importance since diallel analysis will then have become an even more powerful technique for assessing the relative merits of inbred lines as prospective breeding material, especially when it is combined with the breeder's own practical experience. 


\section{SUMMARY}

The interpretation of the results from a diallel cross is based on and subject to certain assumptions. Except for the presence of gene interactions, little is known in practice about the effects on the analysis, should any of these assumptions fail for a particular cross. An investigation was therefore carried out with 8 inbred lines of D. melanogaster to determine the effects of correlated gene distributions on the analysis. Since there were no gene interactions present, the results could be selected in such a way as to display to the full the disturbances which are caused by correlated gene distributions. Associated distributions produce a significant upward curvature on the $\mathrm{Wr} / \mathrm{Vr}$ graph as detected both by the graph itself and subsequent multiple regression analysis. Dispersion, on the other hand, does not give any distinctive curvature to the graph. Analysis not only confirms this, but also shows that when dispersion occurs there is no longer even a significant regression of $\mathrm{Wr}$ on $\mathrm{Vr}$. In general, correlated gene distributions appear to have little effect on the estimates of the relative proportions of dominant and recessive genes which each parent contains, although difficulties may arise when estimating this in the presence of dispersion. Non-random gene distributions do, however, alter the apparent level of dominance, association resulting in an underestimation and dispersion in an overestimation.

The investigation is being continued to see whether it is possible to devise methods by which the effects of correlated gene distributions can be distinguished from those of non-allelic interactions, a distinction which is of considerable importance from both a theoretical and practical viewpoint.

Acknowledgments.-I am indebted to Prof. K. Mather, D.Sc., F.R.S., Dr J. L. Jinks and Dr J. S. Gale for their helpful advice and criticism throughout and to Miss M. J. Leighton for her technical assistance.

This work has been carried out whilst in receipt of an Agricultural Research Council Studentship.

\section{REFERENCES}

BREESE, E. L., AND MATHER, K. 1957. The organisation of polygenic activity within a chromosome in Drosophila. Heredity, $11,373-395$.

COOKE, P., AND MATHER, K. I962. Estimating the components of continuous variation. II. Genetical. Heredity, 17, $211-237$.

HAYMAN, B. I. 1954. The analysis of variance of diallel tables. Biometrics, 1o, 235-244.

hayman, B. I. 1958. The theory and analysis of diallel crosses. II. Genetics, 43, $63-85$.

JiNks, J. L. 1954. The analysis of continuous variation in a diallel cross of Nicotiana rustica varieties. Genetics, 39, 767-788.

JiNks, J. L. 1955. A survey of the genetical basis of heterosis in a variety of diallel crosses. Heredity, 9, 223-238.

JiNKs, J. L. 1956. The $F_{2}$ and backcross generations from a set of diallel crosses. Heredity, 10, I-3o.

Jinks, J. L., AND haYman, B. I. 1953. The analysis of diallel crosses. Maize Genetics Cooperation News Letter, 27, 48-54.

MATHER, к. 1949. Biometrical Genetics. Methuen \& Co., London. 